\title{
Generating Approximate Geographic Descriptions
}

\author{
Ross Turner, Yaji Sripada and Ehud Reiter \\ Dept of Computing Science, \\ University of Aberdeen, UK \\ \{r.turner,yaji.sripada, e.reiter\}@abdn.ac.uk
}

\begin{abstract}
Georeferenced data sets are often large and complex. Natural Language Generation (NLG) systems are beginning to emerge that generate texts from such data. One of the challenges these systems face is the generation of geographic descriptions referring to the location of events or patterns in the data. Based on our studies in the domain of meteorology we present a two staged approach to generating geographic descriptions. The first stage involves using domain knowledge based on the task context to select a frame of reference, and the second involves using constraints imposed by the end user to select values within a frame of reference. Because geographic concepts are inherently vague our approach does not guarantee a distinguishing description. Our evaluation studies show that NLG systems, because they can analyse input data exhaustively, can produce more fine-grained geographic descriptions that are more useful to end users than those generated by human experts.
\end{abstract}

\section{Introduction}

Disciplines such as environmental studies, geography, geology, planning and business marketing make extensive use of Geographical Information Systems (GIS); however, despite an explosion of available mapping software, GIS remains a specialist tool with specialist skills required to analyse and understand the information presented using map displays. Complementing such displays with textual summaries therefore provides an immediate niche for NLG systems.

Recently, research into NLG systems that generate text from georeferenced data has begun to emerge (Dale et al., 2005; Turner et al., 2006; Turner et al., 2008b; Thomas and Sripada, 2008). These systems are required to textually describe the geographic distribution of domain variables such as road surface temperature and unemployment rates. For example, descriptions such as 'road surface temperatures will fall below zero in some places in the southwest' and 'unemployment is highest in the rural areas' need to be generated by these systems. One of the main challenges such systems face is the generation of geographic descriptions such as 'in some places in the southwest' and 'in the rural areas'. Such a task is challenging for a number of reasons:

- many geographic concepts are inherently vague (see for example (Varzi, 2001) for a discussion on this topic);

- often the underlying data sets contain little explicit geographic information for a generation system to make use of (Turner et al., 2008b);

- as input to a generation system, georeferenced data is often complex, constraints imposed on the output text (such as length) may make the traditional approach to the Referring Expression Generation (REG) problem in NLG of finding a distinguishing description implausible (Turner et al., 2008b).

This paper looks at the problem in the context of work the authors have carried out on summarising georeferenced data sets in the meteorology domain. The main feature of our approach is that geographic descriptions perform the dual function of referring to a specific geographic locations unambiguously (traditional function of REG) and also communicate the relationship between the domain information and the geography of the region (novel function of geographic descriptions).

We present a two staged approach to generating geographic descriptions that involve regions. The first stage involves using domain knowledge (meteorological knowledge in our case) to select a frame of reference and the second involves using constraints imposed by the end user to select values within a frame of reference. While generating geographic descriptions it is not always possible to produce a distinguishing description because of the inherent vagueness in geographic concepts. Therefore, in our case we aim to produce a distinguishing description wherever possible, but more often allow non-distinguishing descriptions in the output text, which approximate the location of the event being described as accurately as possible.

After a short overview of the background in $\S 2$, some empirical observations on geographic descrip- 
tions from knowledge acquisition (KA) studies we have carried out are discussed in $\S 3$. Taking these observations into account, in $\S 4$ we describe how this problem is approached using examples from RoadSafe (Turner et al., 2008b), which generates spatial references to events in georeferenced data in terms of regions that approximate their location. It pays particular attention to the use of different perspectives to describe the same situation and how factors that affect what makes a good reference in this domain are taken into account by the system. In $\S 5$ we present a qualitative discussion of aspects of geographic description from the evaluations of RoadSafe that were carried out, and how this relates to future possible work on this topic.

\section{Background}

Much work on generation of spatial descriptions has concentrated on smaller scale spaces that are immediately perceivable. For example, spatial descriptions have been studied from the perspective of robot communication (Kelleher and Kruijff, 2006), 3D animation (Towns et al., 1998) and basic visual scenes (Viethen and Dale, 2008; Ebert et al., 1996). In a more geographical context route description generation systems such as (Dale et al., 2005) and (Moulin and Kettani, 1999) have had wide appeal to NLG researchers. (Varges, 2005) also generate landmark based spatial descriptions using maps from the map task dialogue corpus.

RoadSafe is an NLG system that has been operationally deployed at Aerospace and Marine International (AMI) to produce weather forecast texts for winter road maintenance. It generates forecast texts describing various weather conditions on a road network as shown in Figure 1.

The input to the system is a data set consisting of numerical weather predictions (NWP) calculated over a large set of point locations across a road network. An example static snapshot of the input to RoadSafe for one parameter is shown in Figure 2. The complete input is a series of such snapshots for a number of parameters (see (Turner et al., 2008b) for details).

In applications such as RoadSafe, the same geographical situation can be expressed in a variety of different ways dependent upon the perspective employed, henceforth termed as a frame of reference. Space (geographic or otherwise) is inherently tied to a frame of reference that provides a framework for assigning different values to different locations in space. For example, locations on Earth's surface can be specified by latitude and longitude which provide an absolute frame of reference for geographic space. Cardinal directions such as $\{$ North, East, West and South $\}$ provide an alternative frame of reference for geographic space. As was noted in (Turner et al., 2008b), characterising the data in terms of frames of reference is important because often the only geographic information input data contains are coordinates (latitude and longitude), while the
Overview: Road surface temperatures will fall below zero on all routes during the late evening until around midnight.

Wind (mph): NE 15-25 gusts 50-55 this afternoon in most places, backing NNW and easing 10-20 tomorrow morning, gusts 30-35 during this evening until tomorrow morning in areas above 200M.

Weather: Snow will affect all routes at first, clearing at times then turning moderate during tonight and the early morning in all areas, and persisting until end of period. Ice will affect all routes from the late evening until early morning. Hoar frost will affect some southwestern and central routes by early morning. Road surface temperatures will fall slowly during the evening and tonight, reaching zero in some far southern and southwestern places by 21:00. Fog will affect some northeastern and southwestern routes during tonight and the early morning, turning freezing in some places above $400 \mathrm{M}$.

Figure 1: RoadSafe forecast text showing geographic descriptions underlined

output texts are required to employ a wider choice of frames of reference such as altitude, direction, coastal proximity and population. In RoadSafe the frames of reference employed are always absolute according to Levinson's terminology (Levinson, 2003).

Because the geographic descriptions in RoadSafe do not fit the traditional formulation of the REG problem as finding the most distinguishing description, the most pressing question to address is what makes an adequate reference strategy in this case? This is of course a difficult question and is reliant to a large extent on the communication goal of the system. This paper looks into this problem in the context of the RoadSafe application, that uses a simple spatial sublanguage to generate the types of descriptions required in this application domain.

\section{Observations on geographic descriptions from the weather domain}

In this section we summarise some empirical observations on how meteorologists use geographic descriptions in weather forecasts. It describes work carried out over the course of the RoadSafe project involving knowledge acquisition (KA) studies with experts on summarising georeferenced weather data, observations from data-text corpora (one aimed at the general public and one aimed at experts) and a small study with people from the general public. During RoadSafe we built two prototype georeferenced data-to-text systems that summarised georeferenced weather data: one that produces pollen forecasts based on very simple data (Turner et al., 2006), and the RoadSafe system, which 


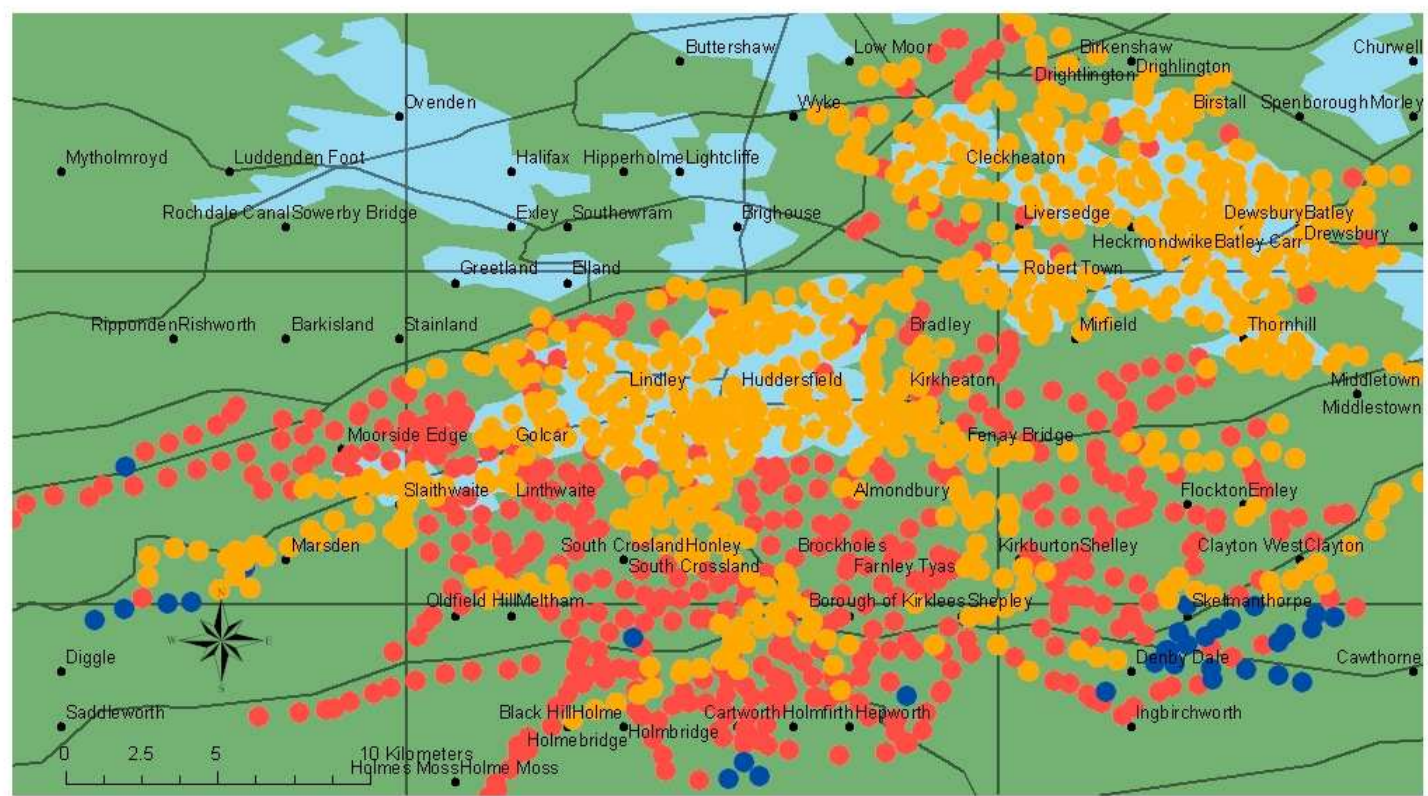

Road Surface Temperatures at 21:00 22/03/08
- marginal
- near critical
- zero

Figure 2: Input data for 'reaching zero in some far southern and southwestern places' in Figure 1

generates road ice forecasts based on complex data. Small corpora consisting of forecast texts and their underlying NWP data were collected in both application domains. Using techniques described in (Reiter et al., 2005) these corpora have been analysed to understand the experts' strategies to describe georeferenced data.

The major finding from our studies is the fact that experts tailor their geographic descriptions to the task context. Not only does the geographic knowledge of the end user have to be taken into account in their descriptions, but also how the geography of the region causes events and patterns in the data. The latter consideration has a large affect on the frame of reference experts employ to describe particular geographic situations. $\S 3.1$ looks at these observations from the point of view of end users of weather forecasts, while $\$ 3.2$ looks at the descriptive strategies of experts.

\subsection{End users' geographic knowledge}

It is a well known and accepted fact that geographic knowledge varies greatly between individuals. To illustrate this point 24 students of a further education college in Scotland were asked a geography question, without reference to a map. Which of four major place names in Scotland (Ayr, Glasgow, Isle of Arran and Stirling) did they consider to be in the south west of the country? The responses showed a great variation in the subjects' geographic knowledge. Half of all sub- jects considered Glasgow and Ayr to be in the south west, one third considered Stirling to be in the south west and most surprisingly only four considered this to be true of the Isle of Arran. The results of this study are surprising because Stirling is the least south westerly place in the list while Isle of Arran is the most south westerly. This study actually agrees well with the studies in psychology on variation in individuals' mental representation of their geographic environment (Tversky, 1993).

Contrast this with the detailed knowledge of a road engineer who the RoadSafe texts are intended for. Road engineers rely upon a large amount of local geographic knowledge and experience when treating roads. Indeed, their spatial mental models are specified at a much finer detail. For example, they get to know where frost hollows tend to form and also come to learn of particular unexpected black spots, such as where garages allow hose water to cover part of a road during winter. This is an important point to be taken into account when communicating georeferenced data as geographic descriptions should be sensitive to that knowledge because it dictates how accurately they will be interpreted by the end user.

Both task context and structural features of data (e.g. number of observations, granularity of measurement), as well as functional features of data (how the entities being described function in space) influence how it is 
described geographically. Analysis of a small pollen forecast corpus (Turner et al., 2006) revealed that forecast texts, contain a rich variety of spatial descriptions for a location despite the data containing only six data points for the whole of Scotland. In general, the same region could be referred to by its proper name e.g. Sutherland and Caithness, by its relation to a well known geographical landmark e.g. North of the Great Glen, or simply by its geographical location on the map e.g. the far North and Northwest. In other words, experts characterise the limited geographic information contained within the data according to the task context. As the consumers of such forecasts are the general public, there is a greater onus on the expert to make the texts more interesting, unlike more restricted domains such as marine (see (Reiter et al., 2005)) or road ice forecasts that require consistent terminology.

\subsection{Experts' descriptive strategy}

Work in psychology has suggested that meteorologists use a dynamic mental model to arrive at an inference to predict and explain weather conditions (Trafton, 2007). Vital to this process is also their ability to take into account how the geography of a region influences the general weather conditions. Understanding the weathers interaction with the terrain enables them to make reliable meteorological inferences particularly when a certain pattern in the data may appear random. It is often unfeasible for a human forecaster to spend large amounts of time inspecting every data point in a detailed visual display. Using experience and expertise a forecaster can use her mental model to 'play out different hypothetical situations' (Trafton, 2007, p.2) and thus arrive at a plausible explanation for an apparently random weather pattern. Consider the following example description of a weather event by an expert taken from our road ice corpus:

- 'exposed locations may have gales at times.'

This is a good example of a forecaster using her meteorological expertise to make an inference about a random weather pattern. Clearly there is no way from inspection of a map one can ascertain with certainty where the exposed locations are in a region. However, an expert's knowledge of how the referent entity (the wind parameter) is affected by geographical features allow her to make such an inference. These pragmatic factors play a large part in determining an experts descriptive strategy, where certain frames of reference may be considered more appropriate to describe certain weather events (Turner et al., 2008a). This comes from weather forecasters' explicit knowledge of spatial dependence (the fact that observations points in georeferenced data at nearby locations are related, and the values of their non-spatial attributes will be influenced by certain geographical features). This is one of the most important and widely understood fact about spatial data from an analysis point of view, and one of the main reasons that it requires special treatment in comparison to other types of non-spatial data. This fact is most clearly outlined by an observation made in (Tobler, 1970, p.3) that 'everything is related to everything else, but near things are more related than distant things'. This is commonly known as the first law of geography and still resonates strongly today amongst geographers (Miller, 2004). The implication of Tobler's first law (TFL) is that samples in spatial data are not independent, and observations located at nearby locations are more likely to be similar. Recasting this into meteorological terms, exposed locations are more likely to be windier and elevated areas colder for example.

In fact, an analogy can be drawn between how meteorologists consider perspectives in their descriptive strategy and the preferred attribute list in the seminal work on REG by (Dale and Reiter, 1995). In their specification of an algorithm for generating referring expressions content selection is performed through the iteration over a pre-determined and task specific list of attributes. In our context, preferred attributes are replaced by preferred frames of reference. This means describing georeferenced data requires situational knowledge of when to apply a particular frame of reference given a particular geographic distribution to describe.

The most striking observation about the expert strategy is that the geographic descriptions in the corpora are approximations of the input (Turner et al., 2008a). The input is highly overspecified with 1000 s of points for a small forecast region, sampled at sub hourly intervals during a forecast period. Meteorologists use vague descriptions in the texts to refer to weather events such as:

- 'in some places in the south, temperatures will drop to around zero or just above zero.'

There are a number of reasons they use this descriptive strategy: the forecasts are highly compressed summaries, as a few sentences describes megabytes of data; very specific descriptions are avoided unless the pattern in the data is very clear cut; experts try to avoid misinterpretation, road engineers often have detailed local geographic knowledge and experts may not be aware the more provincial terminology they use to refer to specific areas. The following section demonstrates how the problem of generating such descriptions is addressed in RoadSafe.

\section{Generating Approximate Geographic Descriptions}

In its current form, where summaries are meant to give a brief synopsis of conditions to the user, RoadSafe follows the approach taken by forecasters as discussed previously. This is unconventional in comparison to traditional REG approaches that aim to rule out all distractors in the domain (properties that are not true of the referent). In a description such as 'reaching zero 
in some places above 100M by 16:00' above, distractors can be defined as the set of points above $100 \mathrm{M}$ that do not satisfy the premise that temperatures will drop below zero. More succinctly, these can be defined as false positives. In fact, the problem can be formulated as a trade off between false positives and false negatives, where false negatives constitute points that are wrongly omitted from the description. For road gritting purposes, costs can be assigned to each type of error: road accidents in the case of false negatives and wasted salt in the case of false positives. As the task dictates, with the higher associated cost it is imperative that a referring expression eliminates all false negatives. Ideally a truly optimal description should then seek to minimise false positives as far as possible, thus reducing the overall cost for the reader. While reducing errors descriptions should also be meteorologically correct, as discussed in the previous section. Using certain frames of reference in certain contexts may result in a poor inference about a particular weather situation (Turner et al., 2008b).

Given this domain knowledge, we can formulate constraints for what makes a good approximate geographic description in this task context:

1. Meteorological correctness (inferencing about causal relationships).

2. Minimise false positives.

3. Complete coverage of the event being described (no false negatives).

These constraints have been realized in a two staged approach to generating geographic descriptions. The first stage involves using domain knowledge (meteorological knowledge in our case) to select a frame of reference, while the second accounts for end-user constraints to select values within that frame of reference. Before we describe the individual stages, two necessary pre-processing stages for generation are described.

\subsection{Geographic characterisation}

As noted in $\$ 2$, observations in georeferenced data often contain little explicit geographic information apart from their coordinates. Geographic characterisation is responsible for assigning a set of qualitative descriptors to each observation based upon a set of reference frames, such that observations can be collectively distinguished from each other. This provides both a criterion for partitioning the data, and a set of properties to generate geographic descriptions. A frame of reference in this context consists of a set of descriptions based upon a common theme such as coastal proximity e.g. $\{$ inland,coastal $\}$ or population e.g. $\{$ urban,rural $\}$. In RoadSafe four frames of reference have been implemented: altitude, coastal proximity, population and direction. Those that make use of human (population) and physical geographical features (altitude, coastal Proximity) can be represented by existing GIS data sets; therefore, in these cases geographic characterisation is simply responsible for mapping observation coordinates to areas of these data sets. In contrast, directions are abstract and require definition. In RoadSafe, geographic characterisation maps each observation to a set of directional areas with crisp boundaries, described in the following section.

\subsection{Pattern formation}

To generate descriptions, the geographic distribution of the event to be communicated has to be approximated using data analysis techniques such as clustering. While not new to data-to-text systems, the novel aspect here is that the data is partitioned based upon the frames of reference that make up the spatial sublanguage of the system. This process summarises the location of the event by measuring its density within each frame of reference's set of descriptions. An example of such a distribution is shown in Figure 3.

$\begin{array}{lll}\begin{array}{lll}\text { Reference Frame } \\ \text { Altitude }\end{array} & \text { Description } & \text { Proportion } \\ & & \\ & \text { 100M } & 0.033 \\ & \text { 200M: } & 0.017 \\ & 300 \mathrm{M} & 0.095 \\ \text { Direction } & 400 \mathrm{M} & 0.042 \\ & & \\ & \text { SSE } & 0.037 \\ & \text { SSW } & 0.014 \\ & \text { WSW: } & 0.048 \\ & \text { TSE } & 0.489 \\ \text { Population } & \text { TSW } & 0.444 \\ & & \\ & \text { Rural: } & 0.039\end{array}$

Figure 3: Density of zero temperatures in Figure 2

While the descriptions within each frame of reference with human and geographical features are dictated by the granularity of available GIS data sets (altitude resolution for example), the boundaries of directional areas require definition. In RoadSafe, because some flexibility in the generated geographic descriptions is desirable, the system uses a four by four grid to split the domain into sixteen equally sized directional areas defined by their their latitude longitude extents. This configuration is shown below where $\mathrm{T}$ stands for true and $\mathrm{C}$ for central in this case:

\begin{tabular}{|c|c|c|c|}
\hline TNW & NNW & NNE & TNE \\
\hline WNW & CNW & CNE & ENE \\
\hline WSW & CSW & CSE & ESE \\
\hline TSW & SSW & SSE & TSE \\
\hline
\end{tabular}

Using a simple set of adjacency matrices based on this grid, RoadSafe represents a set of descriptions depicting the traditional eight main points of the compass plus a further five that we term gradable (central, far south, far north, far east and far west). Alternative con- 
figurations using a greater number of gradable descriptions are possible. These matrices are used by the microplanner to choose attributes to refer to events using the direction frame of reference. One example matrix for each category of directional description are listed below. In each matrix a value of 1 indicates that the event has a non-zero density in that area.

\section{Gradable}

- Far South:

$$
\{T S W, S S W, S S E, T S E\}=\left[\begin{array}{cccc}
0 & 0 & 0 & 0 \\
0 & 0 & 0 & 0 \\
0 & 0 & 0 & 0 \\
1 & 1 & 1 & 1
\end{array}\right]
$$

\section{Intercardinal}

- South West:

$$
\{T S W, W S W, S S W, C S W\}=\left[\begin{array}{cccc}
0 & 0 & 0 & 0 \\
0 & 0 & 0 & 0 \\
1 & 1 & 0 & 0 \\
1 & 1 & 0 & 0
\end{array}\right]
$$

\section{Cardinal}

- South:

$$
\text { SouthEast } \cup \text { SouthWest }=\left[\begin{array}{cccc}
0 & 0 & 0 & 0 \\
0 & 0 & 0 & 0 \\
1 & 1 & 1 & 1 \\
1 & 1 & 1 & 1
\end{array}\right]
$$

In what follows we describe how our two stage strategy is implemented in our system.

\subsection{Frame of reference selection}

The main content selection decision made by the document planner is the choice of which frame of reference to describe a specific weather event such as wind gusts increasing or road surface temperature falling below zero. This decision is based upon both the location of the event as discussed previously, and situational knowledge stored in the knowledge base of the system. Frames of reference where all descriptions have nonzero densities are not considered. Situational knowledge consists of the probability of using each frame of reference given the context (the weather parameter to describe), and is based on corpus frequencies. Rather than simply choosing the frame of reference with the highest density, weighting each frame of reference in this way ensures meteorological correctness as far as possible.

\subsection{Attribute selection}

Once a frame of reference has been selected the microplanner maps the descriptions to abstract syntax templates. As this is fairly trivial for most frames of reference in RoadSafe, because they contain a limited number of descriptions, we will provide an example how this is accomplished for directional descriptions. The input to the microplanner is a structure comprised of the density of the event within the containing area plus its associated adjacency matrix as shown in Figure 4.

$\begin{array}{cc}\text { Location } & \begin{array}{c}\text { Pointratio : } \\ \text { Relation : }\end{array} \\ \text { Container }: & {\left[\begin{array}{cccc}0 & 0 & 0 & 0 \\ 0 & 0 & 0 & 0 \\ 1 & 0 & 0 & 0 \\ 1 & 1 & 1 & 1\end{array}\right]}\end{array}$

Figure 4: REG input to describe Figure 2

The attribute selection algorithm is based upon four constraints incorporating the first two principles of the descriptive strategy outlined at the beginning of this section. They are:

1. Minimise false positives - The description describing the distribution should introduce the least number of distractors. For the above example distribution the set $\{$ South $\}$ ensures coverage but introduces three distractors: CSW, CSE and ESE. While the set of directions \{Far South, South West $\}$ only introduces one: CSW. In general, a measure of how distinguishing a description $x$ is of a distribution $y$ is given by:

$$
\operatorname{distinguishing}(x, y)=\frac{|x \cap y|}{|x|}
$$

Thus, for a distribution $z$ and descriptions $x$ and $y$, $x$ is a more distinguishing description of $z$ than $y$ iff $\operatorname{distinguishing}(x, z)>\operatorname{distinguishing}(y, z)$.

2. Coverage (no false negatives) - The description should completely describe the distribution. The set of directions $\{$ Far South,South West $\}$ completely describes the above example distribution while \{Far South $\}$ does not. For the set of directions $x$ and distribution $y$, the predicate cover $s(x, y)$ is true iff

$$
\frac{|x \cap y|}{|y|}=1
$$

3. Brevity - The set of directions should yield the shortest description of the distribution. For the above example distribution there is only one set of directions that ensures complete coverage. But when faced with a choice for example $\{$ South $\}$ and $\{$ South West, South East $\}$ brevity constraint favours $\{$ South $\}$. In general,the set $x$ should be chosen over $y$ because it is a shorter description. For the distribution $z$ and sets of directions $x, y$ with equal coverage of $z, x$ is a shorter description of $z$ than $y$ iff $|x|<|y|$.

4. Ordering: If two descriptions have equal coverage, cardinality and are equally distinguishing for a 
given distribution, a description is chosen based upon a predefined preference ordering. Each type of property is assigned a score: Cardinal $=3$, Intercardinal $=$ 2 and Gradeable $=1$. Therefore, the set of directions $\{$ Far South, South West $\}$ would be assigned a value of 3.

In classification terms, the first constraint can be considered as precision and the second as recall. The algorithm firstly ranks each individual description in the set described in $\S 4.2$ according to the constraints outlined above. If a single directional term cannot be used to describe the distribution it then incrementally tries to find the highest ranking combination of directions that satisfy the coverage constraint and do not cover the whole region; otherwise, the algorithm terminates by returning the empty set. So, for the example input provided at the beginning of this section it would return the abstract syntax template shown in Figure 4. Quantifiers are selected by applying a simple threshold to the point ratio (which is recalculated should distractors be introduced): some $=>0$, many $=>0.5$, most $=>0.7$. This would be realised as 'in some far southern and southwestern places'.

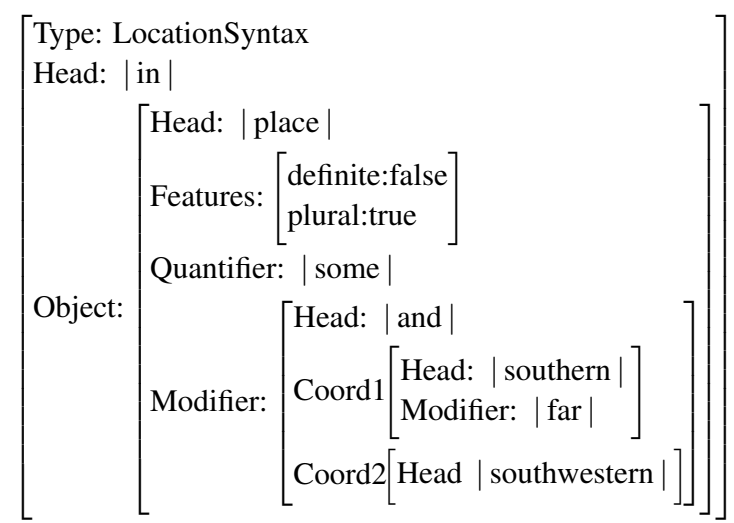

Figure 5: Phrase syntax for input in Figure 4

\section{Evaluation and Discussion}

RoadSafe has been evaluated in post-edit evaluations with meteorologists at AMI and by asking potential users to compare the quality of the summaries to corpus texts based on the same data. While evaluations have been intended to test the overall quality of the texts we have received much feedback on the geographic descriptions the system generates. We have also carried out some comparison of the direction descriptions to those in the corpus, by annotating the corpus descriptions with our adjacency matrices and running them through the system. Descriptions were compared by calculating the Jaccard coefficient between the two matrices. Overall the mean score was 0.53 , with a fairly low perfect recall percentage of $30 \%$. The low precision score is perhaps not surprising as the descriptions generated by RoadSafe are crisp and the corpus descriptions are not solely based on the input data we have available. However, the majority (67\%) of partial alignments were the result of RoadSafe producing a subset of the human desciprition, e.g. northwest versus north, which indicates the system descriptions are more fine grained. In terms of the human descriptions, what was most apparent from this evaluation is the fact that they almost exclusively used the eight major points of the compass.

In terms of feedback experts have commented that generally the location descriptions generated by the system are accurate but should be more general. Of 97 post edited texts generated by the system $20 \%$ of the geographic descriptions were edited.

Most notable was feedback from twenty one road maintenance personnel, who participated in an experiment asking them to compare expert written texts to RoadSafe generated texts based on the same five data sets. The details of this experiment are to be published elsewhere; however, one of the main reasons they gave for liking the style of the generated texts was because they contained more geographic descriptions than the corresponding human ones. The fact that a data-to-text system can analyse every data point is an advantage. In contrast experts have a huge amount of knowledge and experience to draw upon and this reflects in their more general and conservative approach in their geographic descriptions. Perhaps one of their biggest criticisms of the system as a whole is that it doesn't do a good job of generating geographic descriptions that involve motion, such as 'a band of rain works east across the area'. Indeed, this was the most edited type of generated phrase during the post-edit evaluation. There has been little work to our knowledge on describing motion in the NLG literature.

There are many aspects of the generation of geographic that haven't been addressed in this paper and warrant further exploration. Particularly at the content level, there is a need to consider how to account for semantic composition effects caused by overlaying frames of reference. Another question that arises is when is it best to use an intensional rather than extensional description. There is also the question of when to use descriptions that involve relations or gradable properties. These are all choices that a data-to-text system can make that will affect how the summary is interpreted.

\section{Conclusions}

This paper has described an approach for generating approximate geographic descriptions involving regions in the RoadSafe system, which is based on empirical work carried out in the weather domain. Our strategy takes into account constraints on what constitutes a good reference in the application domain described, by taking into account pragmatic factors imposed by both the task context and the end user. What is most apparent from our empirical studies is that geographic descriptions describing georeferenced data are influenced 
by not only by location but also task context. An important observation based on our evaluation studies is that NLG systems by virtue of their ability to analyse input data exhaustively can generate descriptions that are more useful to end users than those generated by human experts.

\section{References}

R. Dale and E. Reiter. 1995. Computational interpretations of the gricean maxims in the generation of referring expressions. Cognitive Science, 19:233-263.

R Dale, S Geldof, and J-P Prost. 2005. Using natural language generation in automatic route description. Journal of Research and Practice in Information Technology, 37(1):89-105.

C. Ebert, D. Glatz, M. Jansche, R. Meyer-Klabunde, and R. Porzel. 1996. From conceptualization to formulation in generating spatial descriptions. In U. Schmid, J. Krems, and F. Wysotzki, editors, Proceedings of the First European Workshop on Cognitive Modeling, pages 235-241.

John D. Kelleher and Geert-Jan M. Kruijff. 2006. Incremental generation of spatial referring expressions in situated dialog. In Proceedings of ACL06, pages 1041-1048.

S. Levinson. 2003. Spatial language. In Nadel L., editor, Encyclopedia of Cognitive Science, volume 4, pages 131-137. Nature Publishing Group.

Harvey J. Miller. 2004. Tobler's first law and spatial analysis. Annals of the Association of American Geographers, 93(3),:574-594.

B. Moulin and D. Kettani. 1999. Route generation and description using the notions of objects influence area and spatial conceptual map. Spatial Cognition and Computation, 1:227-259.

E. Reiter, S. Sripada, J. Hunter, J. Yu, and I. Davy. 2005. Choosing words in computer-generated weather forecasts. In Artificial Intelligence, volume 67, pages $137-169$.

Kavita E Thomas and Somayajulu Sripada. 2008. What's in a message? interpreting geo-referenced data for the visually-impaired. In Proceedings of INLG08.

Waldo Tobler. 1970. A computer movie simulating urban growth in the detroit region. Economic Geography, 46(2):234-240.

Stuart Towns, Charles Callaway, and James Lester. 1998. Generating coordinated natural language and 3D animations for complex spatial explanations. In Proceedings of the Fifteenth National Conference on Artificial Intelligence, pages 112-119, Madison, WI.

J. Gregory Trafton. 2007. Dynamic mental models in weather forecasting. In Proceedings of the Human Factors and Ergonomics Society 51st Annual Meeting, pages 311-314.
R. Turner, S. Sripada, E. Reiter, and I. Davy. 2006. Generating spatio-temporal descriptions in pollen forecasts. EACL06 Companion Volume, pages 163166.

R. Turner, S. Sripada, E. Reiter, and I. Davy. 2008a. Building a parallel spatio-temporal data-text corpus for summary generation. In Proceedings of the LREC2008 Workshop on Methodologies and Resources for Processing Spatial Language, Marrakech, Morocco.

R. Turner, S. Sripada, E. Reiter, and I Davy. 2008b. Using spatial reference frames to generate grounded textual summaries of georeferenced data. In Proceedings of INLG08.

B. Tversky. 1993. Cognitive maps, cognitive collages, and spatial mental models. In A.U. Frank and I. Campari, editors, Spatial Information Theory, pages 14-24. Springer-Verlag, Berlin.

Sebastian Varges. 2005. Spatial descriptions as referring expressions in the maptask domain. In $E N L G$ 05, Aberdeen, UK.

Achille C. Varzi. 2001. Vagueness in geography. Philosophy \& Geography, 4:1:4965.

Jette Viethen and Robert Dale. 2008. The use of spatial relations in referring expressions. In Proceedings of INLG08, Salt Fork, Ohio, USA. 